\title{
Calyceal Diverticula and Megacalycosis Urographic Diagnosis Complications and Treatment
}

\author{
Saleh Ahmed Akares ${ }^{1 *}$, Ahmed Awad Bahomil2, Ali Mothanna Al-Zubaidi ${ }^{3}$ \\ ${ }^{1}$ Urology Unit, Surgery Department, Faculty of Medicine \& Health Sciences, Aden University, Aden, Yemen \\ ${ }^{2}$ Paraclinic Department, Radiology Unit Faculty of Medicine \& Health Sciences, Aden University, Aden, Yemen \\ ${ }^{3}$ Consultant Intervention Gastroenterology and Hepatology King Khalid Hospital Najran, College of Medicine, Najran University, \\ Najran, Saudi Arabia \\ Email: ^salehahmed99123@gmail.com, ahmedbahomil@yahoo.com,dr_ali26@yahoo.com
}

How to cite this paper: Akares, S.A., Bahomil, A.A. and Al-Zubaidi, A.M. (2020) Calyceal Diverticula and Megacalycosis Urographic Diagnosis Complications and Treatment. Open Journal of Urology, 10, 167-175.

https://doi.org/10.4236/oju.2020.105019

Received: April 9, 2020

Accepted: May 10, 2020

Published: May 13, 2020

Copyright $\odot 2020$ by author(s) and Scientific Research Publishing Inc. This work is licensed under the Creative Commons Attribution International License (CC BY 4.0).

http://creativecommons.org/licenses/by/4.0/

\begin{abstract}
Background: Calyceal diverticulae and megacalycosis are rare congenital anomalies. The majorities are asymptomatic but they can present with complications. Objective: The objective of this study is to describe the clinical presentations, complications, urographic diagnosis of calyceal diverticulae, megacalycosis, and their treatment. Methods: A descriptive study carried out over 8 years period from March 2012 to December 2019. In three big hospital of Aden province, we collect 15 patients aged from seven to 41 years (mean 13.5 years), our patients were 9 female $(60 \%)$ and 6 male (40\%). They diagnosed incidentally with calyceal diverticulae and megacalycosis by contrasted urography, describing the clinical manifestations, localization, complications, and type of treatment. Results: Sixty percent of patients with calyceal diverticulae and megacalycosis were women and (40\%) were men. Clinical manifestations including, dull aching flank and abdominal pain in (40\%), acute renal colic (20\%), recurrent urinary tract infection (33\%), and abdominal pain with a fever of unknown origin in (7\%). Calyceal diverticula and megacalycosis were bilateral in (53\%) and unilateral in $(47 \%)$. In the right kidney were (57\%), and in the left kidney (43\%). In the upper pole of the kidney were (53.3\%), middle pole (33.3\%), and lower pole (13.3\%). Complications occurred in $(47 \%)$ of patients. They including, urinary stones in (71.4\%), hypertension (14.3\%), and delayed renal excretion (14.3\%). Conservative treatment carried out in (73\%) and surgically intervened in (27\%). Conclusions: Calyceal diverticula and megacalycosis are rare anomalies. Dull aching flank pain and recurrent urinary tract infections are the most frequent clinical presentations. The most common complications are urinary stones. Conservative treatment is a common type of treatment.
\end{abstract}




\section{Keywords}

Calyceal Diverticulae and Megacalycosis, Diagnosis, Treatment

\section{Introduction}

Calyceal diverticula and megacalycosis are rare congenital anomalies. Calyceal diverticulae present as a cystic cavity of the renal calyceal system which communicates with it through a narrow fornical cannel, while megacalycosis is unilateral dilatation of the renal calyces in the presence of normal undilated renal pelvis and ureter. It results from abnormal budding of the ureteric bud with subsequent lack of parenchymal induction and resultant cystic cavity formation. Megacalycosis is a rare congenital malformation, characterized by homogenous dilatation of the renal calyces with non-obstructive and non-progressive evolution [1] [2]. Calyceal diverticulae were seen in $0.05 \%$ of patients out of 12,000 of examined excretory urography. In the majority of cases are asymptomatic, and incidentally diagnosed during imaging study but clinically can result in a variety of complications [3] [4]. Upright or right lateral decubitus plain abdominal radiography shows the half-moon shape of milky calcium appearance which is the pathogenic feature [5]. Ultrasound is the first investigation but is inconclusive because it resembles a simple cyst [6] [7]. The diagnosis is made by intravenous urography and computerized tomographic urography in complex cysts [8]. Stones in the pelvicalyceal diverticula occurred in up to $50 \%$ of patients and recurrent urinary tract infections in $25 \%$ of patients [9]. Historically open surgical procedures include excision, marsupialization, diverticulectomy or partial nephrectomy. Extracorporeal Shock wave Lithotripsy (SWL) is considered as the first line for treatment [10]. Percutaneous extraction and diverticulum fulguration with canal dilatation provide a good immediate success rate, and low morbidity, it is now accepted as the standard treatment [11]. The diagnosis of calyceal diverticula is a challenge, especially in children. They can result in various complications and diagnostic difficulties.

The objective of this study is to describe the diagnosis of calyceal diverticulae and megacalycosis and their treatment.

\section{Methods}

A descriptive study carried out over 8 years period from March 2012 to December 2019. In three big hospitals (Al-gamoriah teaching hospital, Al-Naquib and Almansorah Hospital) in Aden province, we collected 15 patients aged from seven to 41 years (mean 13.5 years), our patients were 9 female (60\%) and 6 male (40\%), Sixty percent of patients were children $(60 \%)$ and $(40 \%)$ were adults. They presented with nonspecific clinical manifestations, and incidentally diagnosed with calyceal diverticulae and megacalycosi by contrasted excretory urography.

Describing the clinical manifestations and localization whether unilateral or 
bilateral, poles localization whether in the upper, middle, or lower pole of kidneys, the radiologic findings, complications, and type of treatment whether medical or surgical interventions. The data collection was retrieved from medical outpatients' registries and Hospitals' retained Medical documents.

Inclusion: We included those patients who attended these three Hospitals in Aden Governorate and diagnosed with calyceal diverticulae or megacalycosis.

Exclusion: The patients who had the acquired types, and those diagnosed as simple or complex renal cysts were excluded from the study.

Statistic: Statistics method carried out with frequencies and percentages, and confection of tables carried out manually using a summary measure. Data was processing and analyzed using Social Science Statistics software.

Ethical Approval and Consent for patients were not applicable, and patients' identifiers and organ exposure for patients are not involved.

\section{Results}

Of the total 15 patients included in our study nine patients (60\%) with calyceal diverticulae and megacalycosis were women's and six patients (40\%) were men. The clinical manifestations (Table 1) including dull flank and abdominal pain in six patients (40\%), acute renal colic three (20\%), recurrent urinary tract infection five (33\%), and diffuse abdominal pain associated with fever of unknown origin one patient (7\%). Calyceal diverticulae and megacalycosis were diagnosed by contrasted excretory urography. They were bilateral (Figure 1) in eight (53\%) and unilateral (Figure 2) in seven (47\%). Unilaterally localized in the right kidney four (57\%), and in the left kidney three (43\%). Localized in the upper pole of the kidney eight (53.3\%), middle pole four (33.3\%) and in lower pole two patients (13.3\%) as shown in Table 2. Megacalycosis are localized in the central portion of the kidney, characterized by non-obstructive dilatation of pelvis and conserved renal function (Figure 3), and one patient (7\%) of them presented with an associated right renal agenesis (Figure 4). Complications (Table 3) occurred in seven patients (47\%) including, urinary stones in five patients (71.4\%). Anemia and hypertension in one patient (14.3\%), and delayed renal excretion in another one (14.3\%), Bilateral multiple small renal stones in the lower pole (Figure 5) found in three patients (60\%) and unilateral in two (40\%) of patients with urinary stones. Conservative treatment carried out in 11 patients (73\%) and surgically intervened four (27\%). They including (Table 3) excision of the diverticulum

Table 1. Calyceal diverticula and megacalycosis regarding to the clinical presentations.

\begin{tabular}{ccc}
\hline Clinical presentations & NO & $\%$ \\
\hline Dull aching flank pain & 6 & 40 \\
Acute renal colic & 3 & 20 \\
Recurrent UTI & 5 & 33 \\
Abdominal pain with & & $7 \%$ \\
Fever of unknown origin & 1 & 100 \\
Total & 15 & \\
\hline
\end{tabular}


Table 2. Calyceal diverticula and megacalycosis regarding to the site and kidney poles localization.

\begin{tabular}{cccccc}
\hline Unilateral & 7 & 47 & Upper pole & 8 & 53.3 \\
Right & 4 & 57 & Middle pole & 5 & 33.3 \\
Left & 3 & 43 & Lower pole & 2 & 13.3 \\
Bilateral & 8 & 53 & Total & 15 & 100 \\
Total & 15 & 100 & & & \\
\hline
\end{tabular}

Table 3. Congenital calyceal diverticulae and megacalycosis according to complications and surgical procedures.

\begin{tabular}{cccccc}
\hline Complications & No & $\%$ & Surgical procedures & No & $\%$ \\
\hline Urinary stones & 5 & 71.4 & ESWL & 2 & 50 \\
Hypertension & 1 & 14.3 & Excision & 1 & 25 \\
Delay of excretion & 1 & 14.3 & Diverticulutomy & 1 & 25 \\
Total & 7 & 100 & Total & 4 & 100 \\
\hline
\end{tabular}

ESWL $=$ Extracorporeal shock wave lithotripsy.

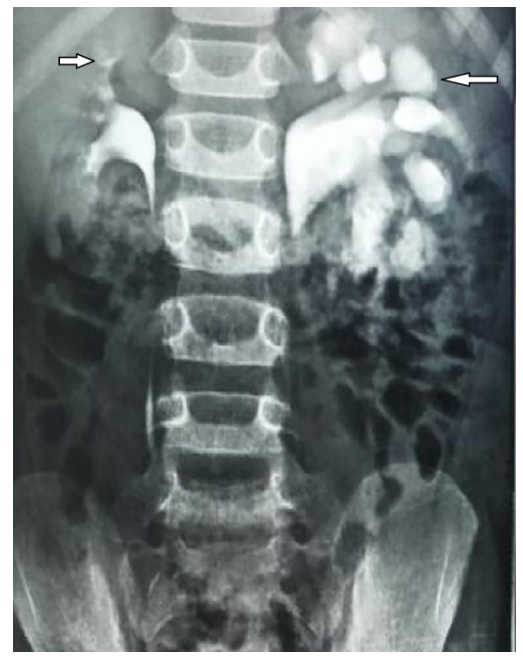

Figure 1. Intravenous urography (IVU) showing bilateral calyceal diverticula, more dilatation in the left side (bilateral arrows).

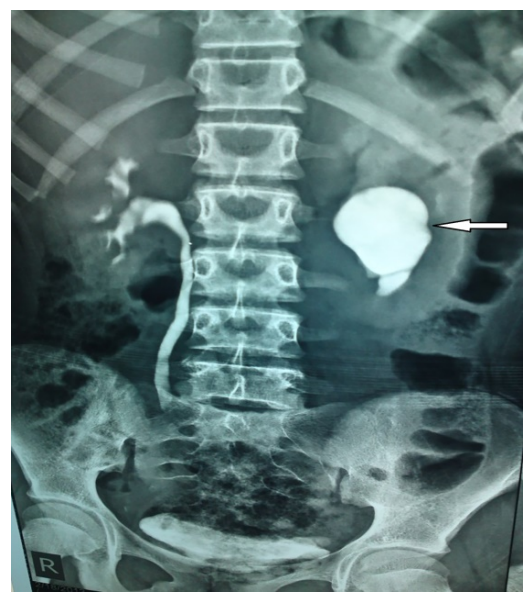

Figure 2. IVU Shows calyceal diverticula in the left pelvicalyceal system (arrow). 


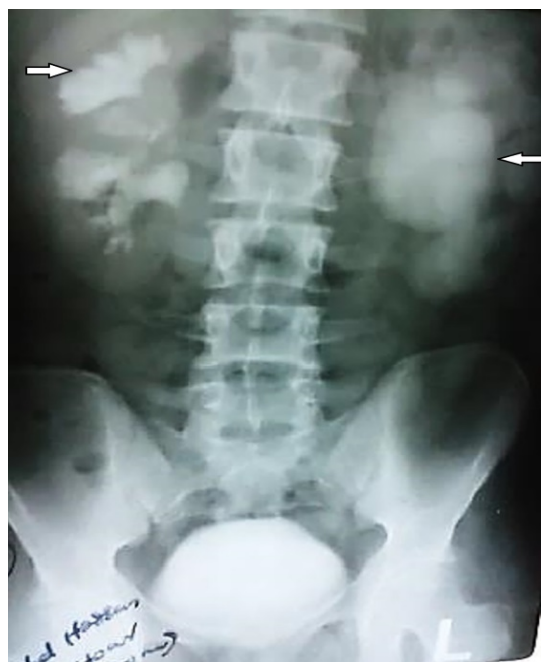

Figure 3. IVU showing bilateral megacalycosis, more calyceal dilatation in the left pelvicalyceal system (bilateral arrows).

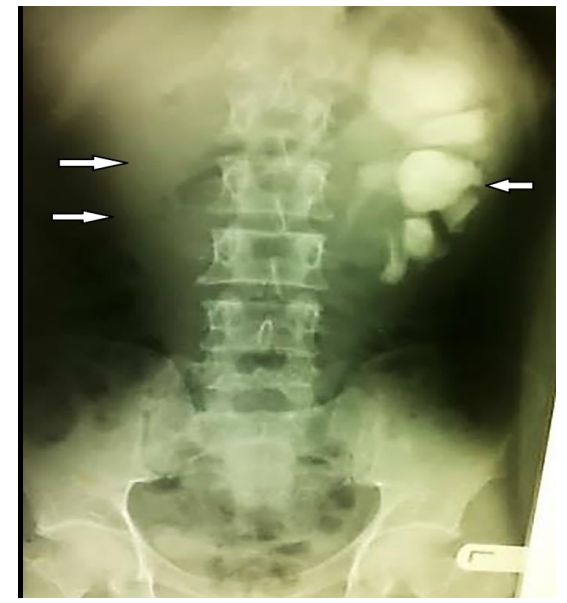

Figure 4. IVU showing left megalocalycosis (Single arrow) associated with right renal agenesis (Two arrows).

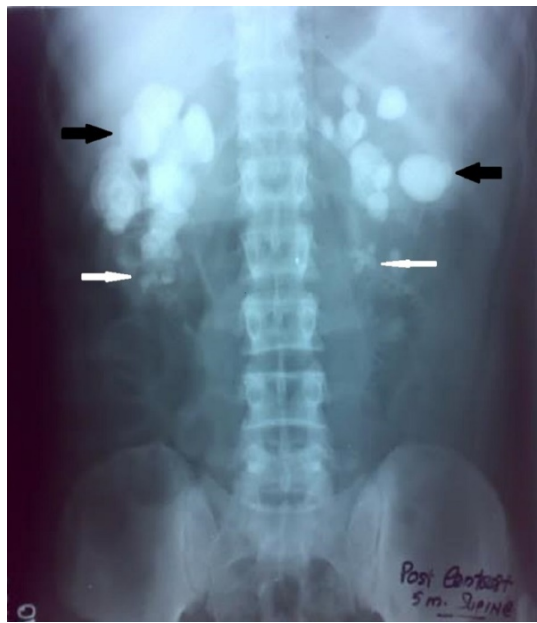

Figure 5. IVU showing bilateral megacalycosis (black arrows) with very small stones in both lower poles of kidneys (white arrows). 
and marsupialization in one patient (25\%), diverticulectomy in another one (25\%), and two patients (50\%) carried out extracorporeal shock wave lithotripsy (ESWL). Stones in the lower poles of kidneys in three patients were very small and treated medically. All patients were under continued follow up at our outpatient clinic and the outcome was excellent.

\section{Discussion}

Calyceal diverticula and megacalycosis are rare congenital anomalies; both represent dilatation of the collecting system but differ in their pathological and radiological features. Two types of calyceal diverticula are recognized. Type I: Calyceal diverticula is the most common, is related to a minor calyx [12]. Type II: It is an extremely rare renal anomaly; it communicates with the renal pelvis or major calyx. It is larger, and symptomatic in the majority of patients. Characterized by non-obstructive dilatation of calyces, the renal pelvis is not dilated, and the ureteropelvic junction is normal and conserved renal function [13]. Calyceal diverticula in the majority are asymptomatic. One-third to one-half of patients present with flank pain, urinary tract infection, and/or hematuria, and stones formation [14]. The calyceal diverticulae were bilateral in 53\% and unilateral in $47 \%$ of patients, Calyceal diverticulae were commonly unilateral and less frequent bilaterally described by Jain et al. 2004 [15]. It is two times more often found in the right kidney than in the left one, and solitary calyceal diverticulae occurs in $90 \%$ of patients described by Surendrababu [16]. They should differentiated simple renal cysts, renal cortical abscess, papillary necrosis, and early stage of adult dominant polycystic kidney disease [17]. Clinical presentations and urographic findings are consistent with reported [18] by Stunnell et al. 2010. Megacalycosis is a rare congenital anomaly, mostly asymptomatic and discovered incidentally or by their complications. The renal pelvis, infundibulum, and ureter are not dilated [19]. Calyces in calyceal diverticulae present as a semilunar configuration rather than the triangular or conical form. Kaviani A. et al. 2010 [20]. Reported a huge extraordinary calyceal diverticulum. In this study stones size range from $3 \mathrm{~mm}$ to $18 \mathrm{~mm}$. Touzani et al. 2015 [21] reported a giant stone of $28 \mathrm{~mm}$ complicating calyceal diverticula, localized in the upper pole of the kidney, treated by flexible uretero-renoscopy. In this study, the majority of patients were treated conservatively in $73 \%$ of patients.

Estrada et al. 2009 described a series of 22 children treated surgically in $43 \%$ of patients. Stones in this study presented in $20 \%$ of patients with calyceal diverticulae less than reported by Estrada et al. 2009 [22]. Waingankar et al. 2014 [23] in his review for a total of 497 patients with calyceal diverticulae determined that they were more common in women in $63 \%$ versus $37 \%$. In men, located in the upper pole of the kidney in $48.9 \%$, middle pole $29.7 \%$ and lower pole in $21.4 \%$, Presented in the right kidney in $49 \%$ and left kidney in $51 \%$, and stones in $96 \%$. Most patients were treated by conservative treatment consistent with this study. Surgery carried out in patients with chronic pain, recurrent or persis- 
tent urinary tract infections, obstructive stones, and renal damage. Surgical treatment is consistent with reported by Nerli R. B. et al. 2014 [24].

Different treatment modalities like percutaneous nephrolitottomy and flexible uretero-renoscopy. Urolithiasis was described by Baso et al. 2015 [25]. Recent study by Laura McGarry concludes that Laparoscopic ablation is the optimal treatment and has significantly higher success rates than the ureteroscopic approach [26]. Carcinoma in a calyceal diverticulum is largely uncommon but some cases were reported [27] this goes with our study no malignancy reported.

\section{Conclusion}

Calyceal diverticulae are rare congenital anomalies and megacalycosis is an extremely rare anomaly. The most common clinical manifestation is abdominal and flank pain in $60 \%$ followed by urinary tract infection. Urinary stones were the most common complications, $73 \%$ of patients treated conservatively and only 27 of patients need intervention.

\section{Conflicts of Interest}

The authors declare no conflicts of interest regarding the publication of this paper.

\section{References}

[1] Kamazyn, B., Kaefer, M. and Jennings, G.G. (2011) Calyceal Diverticula in Pediatric Patients: Spectrum of Imaging Findings. Pediatric Radiology, 41, 1369-1373.

http://link.springer.com/10.1007/s00247-011-2113-4

https://doi.org/10.1007/s00247-011-2113-4

[2] Bawri, B., Puthenveetil, R.T., Baruah, S.J., Barua, S.K. and Bagchi, P.K. (2012) Megacalycosis or Puigvert Disease, a Rare Congenital Calyceal Anomaly: A Report of 3 Cases. UroToday International Journal, 5, Art. 01.

http://www.urotoday.com/index.php?option=com_content\&catid=1181\&id=48656

\&lang=en\&view $=$ article\&Itemid $=0$

https://doi.org/10.3834/uij.1944-5784.2012.04.01

[3] Kavukcu, S., Cakmaci, H. and Babayigiti, A. (2003) Diagnosis of Calyceal Diverticulum in Two Pediatric Patients: Comparison of Sonography, CT and Urography. Journal of Clinical Ultrasound, 31, 218-221. https://doi.org/10.1002/jcu.10154

[4] Mandeville, J.A., Gnessin, E. and Lingeman, J.E. (2013) Percutaneous Management of Calyceal Diverticula: An American Experience. In: Al-Khandari, A.M., Ed., Difficult Cases in Endourology, Springer, London, 33-41.

http://link.springer.com/10.1007/978-1-84882-083-8_4

https://doi.org/10.1007/978-1-84882-083-8_4

[5] Jain, M., Grover, S.B., Kumar, A. and Mahanty, N.K. (2004) Images: Pyelocalyceal Diverticulum. Indian Journal of Radiology and Imaging, 14, 279-281.

http://www.ijri.org/article.asp?issn=0971-3026;year=2004; volume $=14$;issue $=3$; spage $=279$; epage $=281$; aulast $=$ Jain

[6] Gross, A.J. and Hermann, T.R. (2007) Management of Stones in Calyceal Diverticulum. Current Opinion in Urology, 17, 136-140.

http://journals.lww.com/00042307-200703000-00012 
https://doi.org/10.1097/MOU.0b013e328011bcd3

[7] Matalga, B.R., Miller, N.I. and Terry, C. (2007) The Pathogenesis of Calyceal Diverticula Calculi. Urological Research, 35, 35-40.

https://www.researchgate.net/publication/6530498_The_pathogenesis_of_calyceal_ diverticular_calculi https://doi.org/10.1007/s00240-007-0080-x

[8] Radman, J. and Neeb, A. (2005) Congenital Megacalycosis: A Forgotten Diagnosis? Urology, 65, 384-385. https://doi.org/10.1016/j.urology.2004.09.058 https://linkinghub.elsevier.com/retrieve/pii/S0090429504011276

[9] Krambeck, A.E., Leroy, A.J., Fisher, D. and Segura, J.W. (2004) Multiple Calcium Phosphate Stones Occupying a Calyceal Diverticulum. Journal of Urology, 172, 164-165. http://www.jurology.com/doi/10.1097/01.ju.0000129003.74499.2c https://doi.org/10.1097/01.ju.0000129003.74499.2c

[10] Auge, B.K., Munver, R., Kourambas, J., Newman, G.E. and Preminger, G.M. (2002) Endoscopic Management of Symptomatic Caliceal Diverticula: A Retrospective Comparison of Percutaneous Nephrolithotripsy and Ureteroscopy. Journal of Endourology, 16, 557-563. https://doi.org/10.1089/089277902320913233 http://www.liebertpub.com/doi/10.1089/089277902320913233

[11] Okumura, A., Murakami, K., Yoshida, M., Nagakawa, O. and Fuse, H. (2005) Percutaneous Endoscopic Treatment for the Calyceal Diverticular Calculi. International Urology and Nephrology, 37, 5-8.

http://link.springer.com/10.1007/s11255-004-6076-3 https://doi.org/10.1007/s11255-004-6076-3

[12] Bombiński, P., Warchoł, S., Brzewski, M., Biejat, A., Warchoł, T.D., Krzemień, G., et al. (2015) Calyceal Diverticulum of the Kidney-Diagnostic Imaging Dilemma in Pediatric Patients-A Case Report. Polish Journal of Radiology, 80, 27-30. https://www.ncbi.nlm.nih.gov/pmc/articles/PMC4298278/ https://doi.org/10.12659/PJR.892053

[13] Kalaitzis, C., Patris, E., Deligeorgiou, E., Sountoulides, P., Bantis, A. and Touloupids, S. (2015) Radiological Findings and the Clinical Importance of Megacalycosis. Research and Reports in Urology, 7, 153-155. https://doi.org/10.2147/RRU.S81519 https://www.dovepress.com/radiological-findings-and-the-clinical-importance-ofmegacalycosis-peer-reviewed-article-RRU

[14] Hamedbarghi, G.A. and Daglighi, M.H. (2007) Urolithiasis Associated with Bilateral Pelvic Diverticula: A Case Report. Archives of Iranian Medicine, 10, 114-117. https://www.mendeley.com/catalogue/612b5849-3acb-3413-8b3e-86f195507900

[15] Surendrababu, N.R. and Govil, S. (2005) Diagnostic Dilemma: Calyceal Diverticulaversus Complicated Cyst. Indian Journal of Medical Sciences, 59, 403-405. https://www.researchgate.net/publication/7565151_Diagnostic_dilemma_Calyceal_ diverticulum_vs_complicated_cyst_1

[16] Turna, B., Raza, A., Moussa, S., Smith, G. and Tolley, D.A. (2007) Management of Calyceal Diverticular Stones with Extracorporeal Shock Wave Lithotripsy and Percutaneous Nephrolithotomy: Long-Term Outcome. BJU International, 100, 151-156. http://doi.wiley.com/10.1111/j.1464-410X.2007.06911.x

[17] Marietti, S., Woldrich, J. and Durbin, J. (2013) Urologic Findings on Computed Tomography of the Abdomen and Pelvis in a Pediatric Population. Journal of Pediatric Urology, 9, 609-612. https://doi.org/10.1016/j.jpurol.2012.05.014 https://linkinghub.elsevier.com/retrieve/pii/S1477513112001404

[18] Stunell, H., Mc Neill, G., Brown, R.F.J., Grainger, R. and Torreggiani, W.C. (2010) 
The Imaging Appearance of Calyceal Diverticulum Complicated by Urolithiasis. The British Journal of Radiology, 83, 888-894. https://doi.org/10.1259/bjr/22591022

[19] Kasap, B., Kavukeu, S., Soylu, A., Turkmaen, M. and Cecil, M. (2005) Megacalycosis: Report of Two Cases. Pediatric Nephrology, 20, 828-830.

https://www.academia.edu/4934652/Megacalycosis_report_of_two_cases https://doi.org/10.1007/s00467-004-1809-1

[20] Kaviani, A., Hosseini, J., Lotfi, B., Valipour, R. and Sadeghian, I. (2010) Disfiguring Abdominal Mass Due to Huge Extraordinary Calyceal Diverticulum. Urology Journal, 7, 284-286. http://journals.sbmu.ac.ir/urolj/index.php/uj/article/view/830/519

[21] Touzani, M.A. and Ziouziou, I. (2019) Giant Stone Complicating Calyceal Diverticulum. The Pan African Medical Journal, 33, 192.

http://www.panafrican-med-journal.com/content/article/33/192/full

https://doi.org/10.11604/pamj.2019.33.192.18077

[22] Estrada, C.R., Datta, S., Schneck, F.X., Bauer, C.A. and Retick, A.B. (2009) Calyceal Diverticula in Children: Natural History and Management. Journal of Urology, 181, 1306-1311. http://www.jurology.com/doi/10.1016/j.juro.2008.10.043 https://doi.org/10.1016/j.juro.2008.10.043

[23] Waingankar, N., Smith, A.D., Okeke, Z. and Hayek, S. (2014) Calyceal Diverticulum: A Comprehensive Review. Reviews in Urology, 16, 29-43. http://www.pubmedcentral.nih.gov/articlerender.fcgi?artid=PMC4004282

[24] Nerli, R.B., Patil, N., Abhijith, S.M. and Hiremath, M.B. (2014) Symptomatic Calyceal Diverticulum: A Case Report. Journal of the Scientific Society, 41, 133-315. http://www.jscisociety.com/text.asp?2014/41/2/133/132863 https://doi.org/10.4103/0974-5009.132863

[25] Bas, O., Ozyuvali, E. and Aydogmus, Y. (2015) Management of Calyceal Diverticulae: Comparison of Percutaneous Nephrolitottomy and Flexible Uretero-Renoscopy. Urolithiasis, 43, 155-161. https://doi.org/10.1007/s00240-014-0725-5 http://www.ncbi.nlm.nih.gov/pubmed/25249328

[26] McGarry, L., Sahadev, R., Hogan, G., Long, C., Otero, H., Srinivasan, A.K., et al. (2020) Calyceal Diverticula in Children: Laparoscopic Marsupialization Is the Optimal Intervention. Journal of Pediatric Urology. https://linkinghub.elsevier.com/retrieve/pii/S1477513120300176 https://doi.org/10.1016/j.jpurol.2020.01.014

[27] Mitome, T., Tabei, T., Tsuura, Y. and Kobayashi, K. (2018) Squamous Cell Carcinoma in a Calyceal Diverticulum Detected by Percutaneous Nephroscopic Biopsy. Case Report in Oncology Medicine, 2018, Article ID: 3508537.

https://doi.org/10.1155/2018/3508537 